\title{
APLICACIÓN DE TÉCNICAS SIG EN LA PLANIFICACIÓN DEL TRANSPORTE POR CARRETERA EN EXTREMADURA (ESPAÑA)
}

\author{
Julián Mora Aliseda ${ }^{1}$ \\ José Manuel Nogales Galán ${ }^{2}$ \\ José Antonio GutiérRez Gallego ${ }^{2}$
}

Tomás CorTés Ruíz

\begin{abstract}
Resumen - En la planificación del transporte se presta un interés creciente al papel que juegan las infraestructuras como elemento que estructura el territorio. Las nuevas carreteras modifican las condiciones de accesibilidad, lo que a su vez repercute en las potencialidades de desarrollo regional. Los Sistemas de Información Geográfica (SIG) constituyen una herramienta muy apropiada para el cálculo de los niveles de accesibilidad y la elaboración de la cartografía de los resultados. El objetivo que este trabajo pretende es la realización de un modelo del transporte en el territorio de la Comunidad Autónoma de Extremadura, mediante la creación de un SIG, para inventariar, analizar y planificar las infraestructuras en el territorio desde un punto de vista económico y social. Los indicadores son calculados con respecto a los núcleos de población de Extremadura más poblados.
\end{abstract}

Palabras claves : SIG, transporte, planificación, accesibilidad.

Resumo - APLICAÇÃo DE TÉCNICAS SIG NO PLANEAMENTO DO TRANSPORTE RODOVIÁRIO NA EXTREMADURA (Espanha). Na planificação do transporte atribui-se cada vez maior importância ao papel que as infra-estruturas desempenham como elemento que estrutura o território. As novas vias rodoviárias modificam as condições de acessibilidade, influenciando assim as potencialidades do desenvolvimento regional. Os sistemas de informação geográfica (SIG) constituem uma ferramenta muito útil para o cálculo dos níveis de acessibilidade e para a cartografia de resultados. O objectivo deste trabalho é a realização de um modelo de transporte para o território da "Comunidad Autonómica de Extremadura», mediante a criação de um modelo SIG, para inventariar, analisar e planificar, de um ponto de vista

\footnotetext{
1 Departamento de Geografía y Ordenación del Territorio. Universidad de Extremadura. E-mail: jmora@unex.es

2 Departamento de Expresión Gráfica. Área de Ingeniería en Geodesia, Cartografía y Fotogrametría. Universidad de Extremadura.
} 
económico e social, infra-estruturas no território. Desta forma, é possível avaliar os efeitos da implantação de infra-estruturas no território, tendo em conta o transporte rodoviário. O cálculo de trajectos mínimos é de grande importância para a realização de modelos SIG realistas em relação à acessibilidade. Estes modelos necessitam do cálculo de distâncias entre pares de objectos. Por meio deste cálculo de trajectos mínimos, podemos obter a medição de distâncias na rede em vez de utilizar distâncias em linha recta. Com a utilização dos SIG é possível implantar modelos que permitam prever tendências futuras. Para estudar as potencialidades de um modelo criado, determinam-se primeiro indicadores que mostram os diversos graus de comunicação entre as localidades da Extremadura num contexto puramente regional. Os indicadores calculados para os núcleos urbanos de Extremadura são a acessibilidade tanto absoluta como relativa, tempos de acesso mínimo e a atracção gravitatória exercida pelos núcleos populacionais Extremenhos mais importantes. Com os resultados obtidos para cada indicador, estamos em condições de gerar os respectivos mapas.

Palavras-chave : SIG, transporte rodoviário, planeamento, acessibilidade.

Summary - GIS In ROAd tRANSPORT PlanNing In Extremadura (SPAin). Increasingly more importance is given to the role played by infrastructures as an element that shapes the landscape. New highways modify conditions of access, which consequently influence potential regional development. In this sense, Geographical Information Systems (GIS) are suitable tools for the calculation of accessibility levels and the implementation of cartographical outputs and results. In the light of these premises, this work is aimed at designing a GIS transportation model in the Regional Community of Extremadura in order to make an inventory and to plan and analyse regional infrastructures from economic and social perspectives. It is also our aim to evaluate the effects derived from infrastructure changes on the landscape by considering the highway transportation systems. In this sense, the calculation of shorter pathways is of great importance in order to achieve realistic accessibility GIS models. These models need calculations of the distance between couples of objects. By means of the calculation of the shortest pathways, distances can be precisely measured through the network that monitors the movements instead of using straight-line distances. By using GIS, models can be created that allow us to predict future trends. In order to study the potential of the designed model, different indicators have been determined showing the communication levels in different areas of Extremadura. Indicators calculated for the different population centres and areas are accessibility in absolute and relative terms, times of minimum access and the gravitational pull in comparison with the most significant populated areas. Maps are then generated with the results obtained from each of the indicators.

Key words : GIS, road transport, planning, accessibility.

Resumé - Application de techniques SIG à la Planification des transports ROUTIERS EN EXTREMADURA (ESPAGNE). Les nouvelles voies routières rapides jouent un rôle croissant dans la structuration des territoires. Elles modifient les conditions d'accès et influencent ainsi les potentialités de développement régional. Les systèmes d'information géographique (SIG) sont un instrument précieux pour calculer et cartographier les niveaux d'accessibilité. On a cherché à réaliser un modèle de 
transport pour l'Extremadura, grâce à un modèle SIG permettant d'inventorier, d'analyser et de planifier les infrastructures économico-sociales de la région. Le calcul des trajets minimaux entre paires d'objets permet de mesurer les distances non pas en ligne droite, mais au long de réseaux. La création de modèles permet de prévoir les tendances futures. Pour tester ces modèles, on a déterminé d'abord les indicateurs des divers degrés de communication entre les localités d'Extremadura. On a pris en compte l'accessibilité, tant absolue que relative, le temps d'accès minimum et l'attraction gravitaire exercée par les principaux centres. Les cartes sont construites à partir de ces données.

Mots-clés : SIG, transport routiers, aménagement, accessibilité.

\section{INTRODUCCIÓN}

El transporte es un proceso productivo que consiste en trasladar mercancías y/o personas de un sitio a otro. La diferencia con las comunicaciones es que estas transmiten a distancia un mensaje con ayuda de distintos medios de comunicación (РотRYкоwski et al., 1982).

En la sociedad actual, el transporte es esencial y indispensable para el funcionamiento socioeconómico y la articulación e integración de pueblos y espacios territoriales, a través de los necesarios flujos de personas y mercancías. La necesidad de su existencia para el resto de actividades es tan importante que su desaparición como sector supondrá la desaparición de la sociedad, al menos tal y como hoy la conocemos (Ruiz Requena, 1992).

Las infraestructuras de transportes, en tanto que modifican las condiciones de accesibilidad, constituyen un elemento clave en las políticas de desarrollo regional. Las nuevas inversiones pueden resolver problemas de congestión pero también pueden orientarse a potenciar regiones menos favorecidas. Esto no quiere decir que las infraestructuras del transporte basten por sí solas para generar el desarrollo regional, de hecho hay casos en que ha ocurrido lo contrario, pero sí es un factor necesario.

Las actuaciones en infraestructura de transportes modifican el marco territorial, ya que producen una reducción de distancias, una concentración del espacio (GutiérRez Puebla, 1994). El espacio geográfico no se concibe como un todo absoluto, sino como un marco en el que las condiciones de accesibilidad se alteran constantemente.

De esta manera los conceptos de accesibilidad y perifericidad están relacionados con la dotación de infraestructuras de transporte. Hay que tener en cuenta que más infraestructura de transportes no significa necesariamente más accesibilidad. Algunas medidas se localizan sobre ejes estructurantes y producen cambios importantes de accesibilidad, pero otras se ubican fuera de esos ejes y los efectos producidos son más débiles. Por esto es necesaria la realización de estudios previos que determinen en qué medida cambian las condiciones de accesibilidad de una región. 
Mediante la utilización de los Sistemas de Información Geográfica (SIG) es posible implementar modelos que permitan predecir cuáles serán las tendencias futuras o qué efectos se producirán en caso de que cambie alguno de los elementos del sistema territorial y así es posible que los planificadores y políticos puedan explorar los posibles escenarios y obtener una idea de las consecuencias de una actuación antes de que se hayan cometidos errores irreversibles.

El objetivo general que se pretende con este trabajo es la creación de un modelo del transporte por carretera en el territorio de la Comunidad Autónoma de Extremadura (fig. 1), mediante el desarrollo de un SIG, para inventariar, analizar y planificar las infraestructuras desde un punto de vista económico y social. No se ha tenido en cuenta el transporte ferroviario y aéreo por el escaso volumen de personas y mercancías que transporta hoy día en Extremadura. El objetivo concreto de este trabajo es determinar la accesibilidad de las poblaciones extremeñas a los principales Centros de Actividad Económica de Extremadura. Para ambos objetivos es necesario formular por un lado un modelo de transportes basado en la teoría de transportes que trate de aproximarse al modelo real todo lo posible y por otro lado hay que aplicar las herramientas adecuadas de los SIG para materializar este modelo y de esta forma poder estudiarlo. Entre ambas fases del trabajo es necesario crear el modelo conceptual mediante el cual se relacionan las entidades del mundo real y sus atributos con las entidades del modelo informático y sus datos.

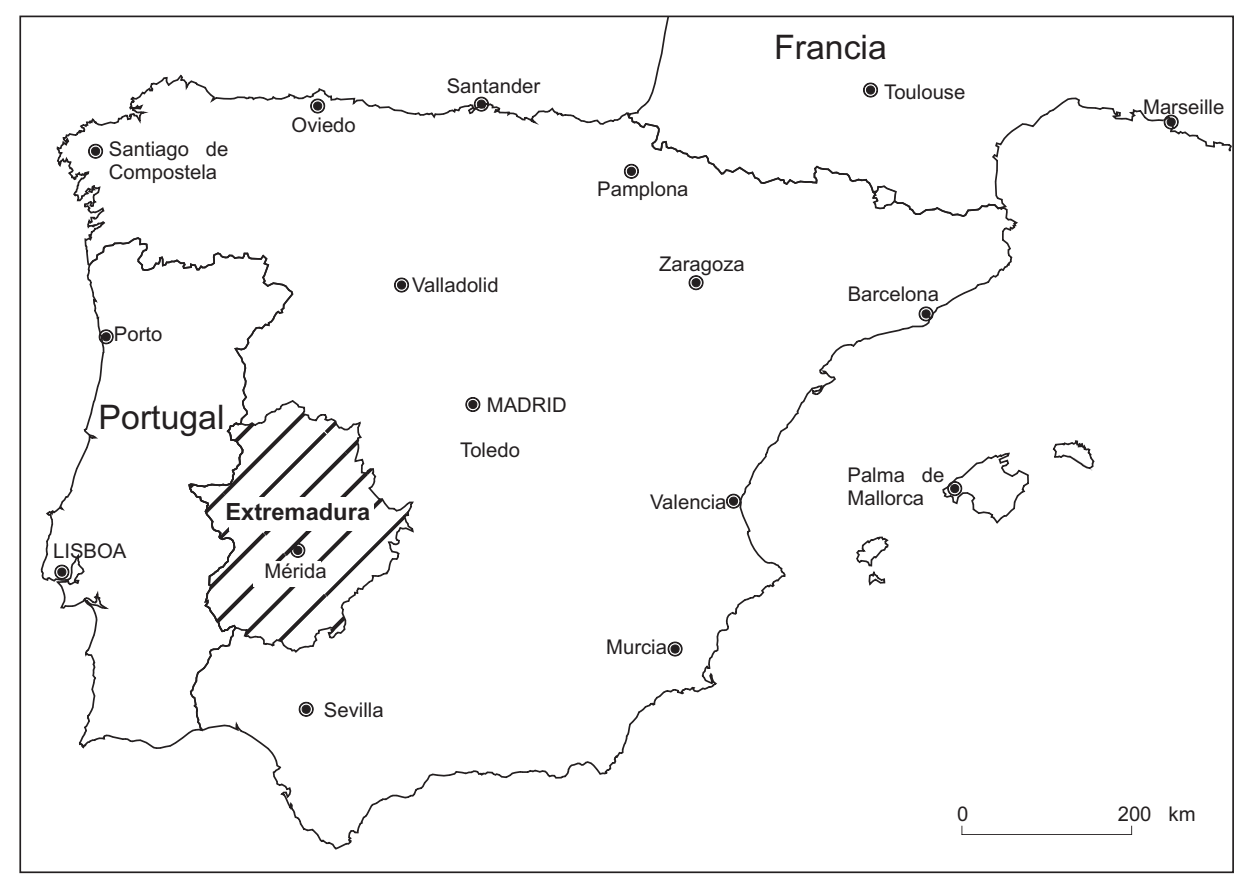

Fig. 1 - Situación de Extremadura en la Península Ibérica.

Fig. 1 - Location of Extremadura in the Iberian Peninsula. 


\section{INDICADORES PARA LA DETERMINACIÓN DE LA ACCESIBILIDAD TERRITORIAL}

Un lugar o una zona del territorio es accesible cuando tiene acceso o, con mayor precisión, cuando este acceso es fácil (Ruiz Requena, 1992). Si lo que queremos es perfilar un concepto técnico y cuantificable la cuestión es mucho más difícil, cada uno de los indicadores que habitualmente se utilizan, miden realmente distintas características del territorio en relación con el sistema de transporte. La accesibilidad permite explicar las posibilidades de interacción entre los distintos puntos de un territorio.

Una aplicación de los índices de accesibilidad absoluta y relativa se pueden ver dentro del estudio de Gutiérrez Puebla et al. (1994). En este estudio se trataba de evaluar los efectos de las actuaciones contempladas en el Plan sobre la accesibilidad territorial, teniendo en cuenta los distintos modos de transporte: carretera, ferrocarril, puertos y aeropuertos.

Una variante de ese estudio es la que realizamos en esta aplicación pero para el caso concreto de Extremadura y con un grado mayor de detalle ya que se han considerado todos los municipios de Extremadura y no sólo los de mayor renta como en el caso del citado estudio.

En el estudio de Gutiérrez Puebla se considera la renta como factor de atracción de los núcleos de actividad económica. En nuestro caso hemos considerado el número de habitantes como factor de aglomeración de las principales ciudades de Extremadura, ya que existe una relación directa entre el número de habitantes y la renta del municipio, al no existir grandes diferencias de renta entre los habitantes de las diferentes localidades o comarcas extremeñas (La CAIXA, 2002).

Los centros de actividad económica de Extremadura con una población superior a 25000 habitantes (Badajoz, Cáceres, Mérida, Don Benito-Villanueva de la Serena, Plasencia y Almendralejo), concentran el 35\% de la población extremeña. Las localidades de Don Benito y Villanueva de la Serena debido a su proximidad geográfica y a su complementariedad se han considerado como un único núcleo de población (Mora et al., 1998).

En el cálculo de los índices de accesibilidad es necesario tener en cuenta la impedancia o la resistencia al movimiento de la red de transportes. Las impedancias constituyen un elemento clave en los análisis de accesibilidad, ya que simulan el efecto de resistencia que se asocia al desplazamiento por al red, y que pueden ser distancia, tiempo, coste, etcétera. En nuestro caso, hemos tenido en cuenta los tiempos mínimos de desplazamiento para ir desde una localidad de Extremadura a cada uno de los centros de actividad económica. Como los tiempos de desplazamiento a través de la red son función de la velocidad específica y calidad de los tramos de carretera, los indicadores propuestos reflejan distintos aspectos de la accesibilidad infraestructural. 
En este estudio se han determinado además de los índices de Accesibilidad Absoluta y Relativa, los de Tiempo de Acceso Mínimo y de Atracción Gravitatoria de cada localidad extremeña con respecto a los citados centros de actividad económica de Extremadura.

\section{Accesibilidad absoluta}

El indicador de accesibilidad absoluta es sensible a la localización geográfica de los núcleos de población, primando a las regiones centrales, lo que resulta fundamental desde el punto de vista del desarrollo regional, en el que la base territorial no pueden obviarse (GutiérRez Puebla et al., 1993).

Se trata de calcular el promedio de las impedancias que separan a cada núcleo de población con respecto a los diferentes centros de actividad económica a través de la red (por el camino de mínima impedancia), considerando la renta de éstos como factor de ponderación, según la expresión siguiente:

$$
I A A_{i}=\frac{\sum_{j=1}^{n}\left(I R_{i j} \times R C A E_{j}\right)}{\sum_{j=1}^{n} R C A E_{j}}
$$

En esta expresión $I A A_{i}$ es el Índice de Accesibilidad Absoluta del nodo $i$; $I R_{i j}$ es la Impedancia Real (tiempo mínimo de desplazamiento) a través de la red entre los nodos $i$ y $j ; R C A E_{j}$ es la renta del centro de actividad económica en destino, en nuestro caso es el número de habitantes de centro de actividad económica. Los nodos $i$ son cada uno de los núcleos de población de Extremadura, a excepción de los seis centros de actividad económica que son los nodos $j$.

\section{Accesibilidad relativa}

El indicador de accesibilidad relativa, en cambio, neutraliza el efecto de la localización geográfica, con el objetivo de resaltar más los efectos de la oferta infraestructural sobre la accesibilidad. Refleja al mismo tiempo los índices de rodeo (estructura geométrica de la red) y el tipo de infraestructura en la accesibilidad a los principales centros de actividad (Gutiérrez Puebla et al., 1993).

Su formulación es la siguiente:

$$
I A R_{i}=\sum_{j=1}^{n}\left(\frac{I R_{i j}}{I I_{i j}} \times R C A E_{j}\right)
$$

donde $I A R_{i}$ es el Índice de Accesibilidad Relativa; $I R_{i j}$ y $R C A E_{j}$ son términos ya conocidos e $I I_{i j}$ es la impedancia ideal. En el caso del transporte terrestre, la impedancia ideal entre el nodo de origen y el centro de actividad de destino es 
la que se obtendría en línea recta por una infraestructura de gran calidad, una autopista o autovía. Así, para cada relación se calcula el cociente $I R_{i j} / I I_{i j}$, que tenderá a 1 cuando la impedancia real se acerque a la ideal, pero irá aumentando a medida que la primera se aleje de la segunda.

\section{Tiempo de acceso mínimo}

Con este indicador se determina el tiempo de acceso mínimo de una localidad al centro de actividad económica más próximo. Como se han determinado para los índices anteriores el tiempo mínimo a través de la red de carreteras desde cada población a los seis centros de actividad económica, ahora se selecciona el menor de ellos.

$$
T A M i=\min \left(I R_{i j}\right) \forall j
$$

Con este indicador se determina, por un lado, la proximidad de cada localidad extremeña al centro de actividad económica más cercano y su facilidad para acceder a los servicios que presta, y por otro, las zonas más alejadas o periféricas de la región.

\section{Atracción gravitatoria}

Los modelos de gravedad basados en la Ley de Newton se han venido utilizando en estudios geográficos desde hace tiempo. La forma de medir la masa depende del objeto del estudio al igual que la distancia (SÁnchez Zabala, 1988). En nuestro estudio, al igual que en los índices anteriores, se ha considerado el número de habitantes como valor de las masas de los núcleos de población y el tiempo de acceso mínimo a través de la red como valor de la distancia entre las poblaciones consideradas. La expresión utilizada ha sido la siguiente:

$$
A G_{i}=\max \left(k \frac{R N_{i} \times R C A E_{j}}{I R_{i j i j}^{2}}\right) \forall j
$$

donde $A G_{i}$ es el Índice de Atracción Gravitatoria de un núcleo de población extremeño $i, R N_{i}$ es el número de habitantes del núcleo de población considerado, $R C A E_{j}$ e $I R_{i j}$ son términos ya conocidos. Como es éste un estudio comparativo asignamos a la constante $k$ un valor igual a 1 . Este índice es muy sensible a la impedancia (tiempo) al estar elevada al cuadrado por los que la fuerza de atracción gravitatoria de un núcleo de población es muchas veces despreciable respecto de los centros de actividad económica lejanos. Por lo que finalmente, la atracción gravitatoria de una localidad se determina seleccionando el valor máximo de las que tiene con respecto a los seis núcleos de actividad económica.

Con este índice se llega a determinar el área de influencia o de atracción gravitatoria de cada centro de actividad económica. 


\section{METODOLOGÍA}

Antes de aplicar este modelo conceptual al modelo teórico hay que hacer unas apreciaciones sobre este último:

- El sistema de transportes regional es un sistema cerrado;

- Los núcleos de población se consideran puntuales, sin superficie.

Todos los enlaces y comunicaciones se realizan dentro del sistema. Se trata de aislar un sistema y estudiarlo por separado, para analizar cuál es su funcionamiento interno (GURRÍA,1992). Es evidente que este sistema se encuentra integrado en otro sistema mayor que sería la Península Ibérica. Desde la integración de Portugal y España en la Unión Europea y la desaparición de la frontera entre ambas naciones, las comunicaciones e intercambios entre estos dos países tiende a crecer sobre todo en una región fronteriza como la extremeña. En un segundo estudio habría que estudiar como interacciona el sistema de transporte extremeño con los de su entorno.

\section{El modelo entidad-relación}

Lo primero para realizar el modelo conceptual es establecer las relaciones entre las distintas entidades que componen el sistema de información mediante el modelo entidad-relación. Se distinguen tres tipos de entidades:

a. Núcleos de población: Los núcleos van a ser los destinos final e intermedios de la ruta seguida para acceder a otro punto del territorio;

b. Nodos: Establecen las intersecciones entre distintas carreteras junto con los núcleos de población;

c. Tramos de carretera: son las distintas partes de la carretera a partir de los nodos.

Cada entidad tiene una serie de propiedades, siendo el código el que identifica de forma única a cada uno de los registros que componen la entidad.

Una vez definidas cada una de las tablas del sistema de información se establecen las relaciones entre las entidades del modelo entidad-relación.

\section{Bases de datos y almacenamiento de la información}

Se diseña el diagrama de flujo de datos de información entre los distintos elementos que componen el sistema de información. Los distintos entes externos que van a dar y recibir información del sistema son: Instituto Nacional de Estadística (INE), Servicios de Carreteras (Diputaciones provinciales de Badajoz y Cáceres, Consejería de Fomento y Ministerio de Fomento), Instituto Geográfico Nacional (IGN), y los usuarios.

La información recibida o generada en el SIG se ubica en dos tipos de almacenes: 
a) unos son los almacenes propiamente dichos donde después de recibir la información esta se almacena (esta información puede ser requerida de nuevo por la misma entidad);

b) y aquellos que sirven de estacionamiento transitorio de los datos (se utilizan sobre todo para la separación estructurada de la información para cada uno de los procesos donde se usa esta información).

\section{PROCEDIMIENTOS PARA IMPLEMENTACIÓN DEL MODELO CONCEPTUAL EN SIG}

Una vez establecidos los almacenes establecemos los procedimientos. Estos procedimientos tienen el fin del cumplimiento del objetivo del Sistema de Información. Con los datos obtenidos de los almacenes los procedimientos manejan la información en función del estudio deseado.

Los procedimientos en nuestro Sistema de Información son: la captura de la información, el tratamiento de la misma y el análisis.

\section{Captura de la información}

Este procedimiento recibe información del IGN y del INE y del SERVICIO DE CARRETERAS, y la almacena en el almacén NÚCLEOS. Se realizan en esta fase dos procedimientos:

a. Digitalización: la entidad externa (IGN) facilita la cartografía, que es digitalizada, a escala 1/200 000 de las provincias de Cáceres y Badajoz;

b. Normalización: la base de datos se estructura en tablas para los núcleos y para las carreteras. En la tabla de núcleos aparece: el nombre del municipio, número de habitantes y coordenadas carto-gráficas del núcleo obtenidas de la digitalización. En la tabla de carreteras figura la denominación, la longitud y velocidad específica de la misma.

\section{Tratamiento de la información}

Aquí recibimos información del procedimiento CAPTURA DE INFORMACIÓN y del almacén NÚCLEOS. La tratamos y determinamos los nodos y como consecuencia los tramos de carretera, almacenando esta información en sus respectivos almacenes NODOS y TRAMOS DE CARRETERA. Este procedimiento se divide a su vez en otros dos:

a. Enlace de información: a la información gráfica enlazaremos la información alfanumérica;

b. Creación de topología: se establecen las relaciones topológicas entre las distintas entidades gráficas. Una vez establecidas las relaciones topológicas las almacenamos en los almacenes NODOS y TRAMOS DE CARRETERAS. 


\section{Análisis}

Se realiza en dos fases:

a. Determinación de los caminos óptimos: se determina el camino de menor duración entre las 380 localidades extremeñas y cada uno de los seis centros de actividad económica a través de la red. De esta manera cada núcleo de población adquiere seis nuevos atributos que se almacenan en NÚCLEOS;

b. Cálculos de índices: se realiza el cálculo de índices de accesibilidad con las expresiones ya expuesta. Se obtienen unos nuevos atributos de cada localidad extremeña: los valores de accesibilidad absoluta y relativa, el tiempo de acceso mínimo y la atracción gravitatoria.

\section{ANÁLISIS DE ACCESIBILIDADES EN EXTREMADURA}

\section{Metodología para la elaboración de la cartografía de accesibilidad}

Los valores de accesibilidad fueron asignados a cada uno de los 380 municipios de Extremadura, considerándolos como elementos puntuales. A partir de aquí se generó un Modelo Digital del Terreno (MDT) para cada uno de los valores de accesibilidad. El método utilizado para la creación de los diversos MDT ha sido el de Inversa de la Distancia Euclídea al cuadrado (IDW). Esta técnica consiste en generar una matriz regular, donde a cada una de las celdas le corresponde el valor de la media ponderada de los seis puntos más próximos a la localización de la celda. La ponderación se realiza de forma inversamente proporcional al cuadrado de la distancia Euclídea de cada punto a la localización de la celda.

La clasificación se ha realizado por el método de rupturas naturales (natural break), que consiste en la agrupación de los valores una vez ordenados de mayor a menor y teniendo en cuenta las repeticiones. Así, la accesibilidad se clasifica en muy buena, buena, normal, mala y muy mala. Se consideró que este método permitía clasificar de forma bastante homogénea todo el territorio extremeño en sus distintos niveles de accesibilidad. No se han definidos unos límites en los intervalos al ser un estudio comparado que depende de la amplitud y de las característica económicas del territorio considerado. Así, en el estudio de Gutiérrez Puebla, sobre el Territorio de la España peninsular, Extremadura era una de las zonas con peor accesibilidad de España al estar alejada de las grandes ciudades españolas y no tener en su territorio ciudades que pudieran considerarse como Centros de Actividad económica españoles.

Con los resultados obtenidos para cada uno de los indicadores se generan los mapas de isoaccesibilidad.

\section{Accesibilidad absoluta}

Como ya se apuntó con anterioridad el indicador de Accesibilidad Absoluta nos muestra el grado de centralidad de una localidad dentro del territorio con- 
siderado. Este indicador se basa en el coste del transporte y en los modelos de potencial de mercado. Por tanto nos muestra el coste potencial del transporte asociado a cada núcleo de población en su relación con los centros de actividad económica.

Existe una amplia zona central en la región (fig. 2), que engloba a casi todos los Centros de Actividad Económica (excepto Plasencia), en el que la accesibilidad absoluta es muy buena. Esta zona está desplazada hacia la frontera con Portugal, por la inducción ejercida por la ciudad de Badajoz, y se prolonga hacia el Noreste gracias a la Autovía N-V que acerca las localidades de esta zona a los Centro de Actividad Económica.

A medida que nos alejamos de esta zona central, casi de una forma concéntrica y bastante regular, va empeorando la accesibilidad absoluta hasta llegar a las zonas con peor accesibilidad en los límites con las regiones españolas, de una manera más desfavorable que en la frontera portuguesa, por el efecto de la ciudad de Badajoz. Así las zonas con mala o muy mala accesibilidad absoluta se ubican: al norte en los límites de Extremadura con Castilla-León, al oeste en el límite regional con Castilla-La Mancha y por último al sur en zonas limítrofes con Andalucía.

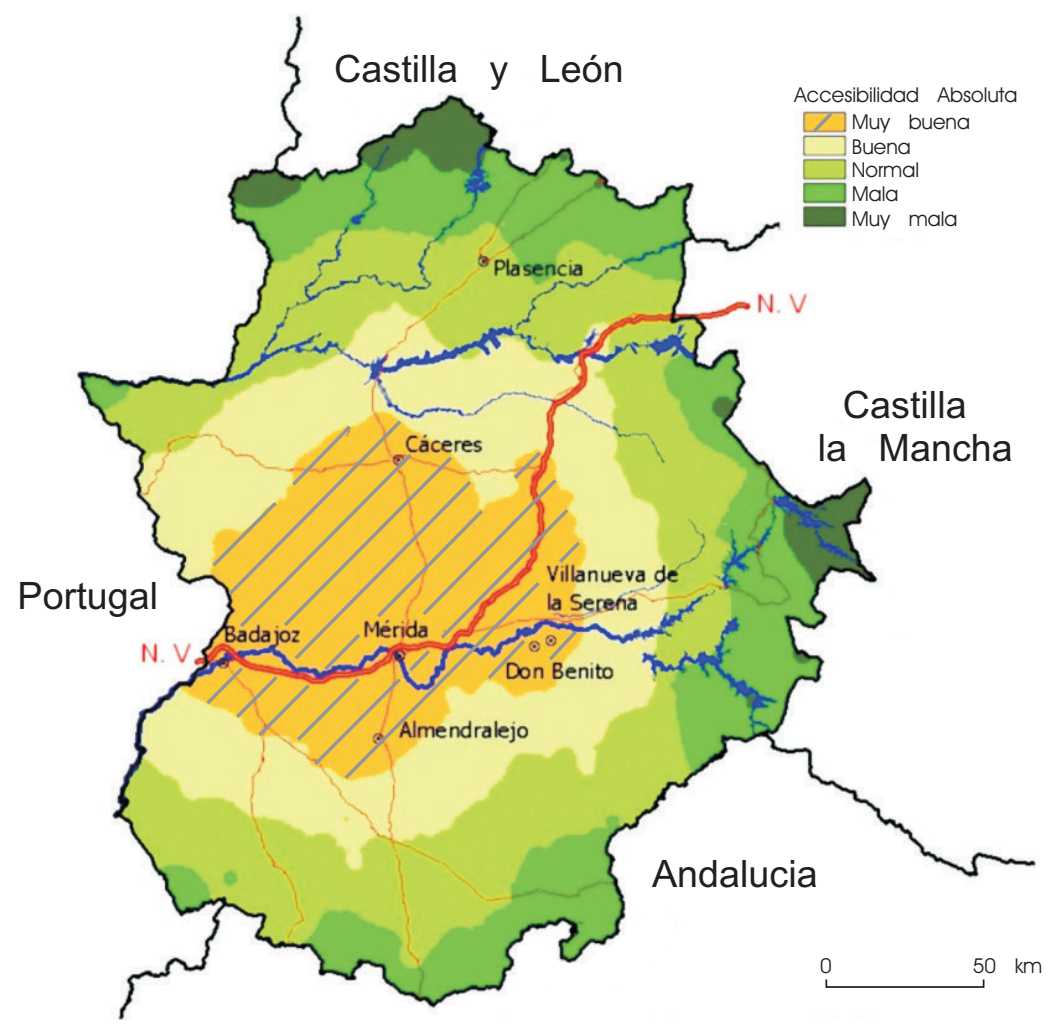

Fig. 2 - Accesibilidad Absoluta en Extremadura.

Fig. 2 - Absolute Accessibility in Extremadura. 


\section{Accesibilidad relativa}

El indicador de Accesibilidad Relativa refleja la distribución espacial de las infraestructuras y su impacto sobre el territorio. Este índice trata de cuantificar la calidad del trazado, es decir su grado de aproximación a la línea recta.

A la vista del mapa correspondiente (fig. 3) se observa que la distribución de los niveles de Accesibilidad Relativa es bastante irregular. A grandes rasgos es mala o muy mala desde el centro de la región hacia la frontera portuguesa y es buena, además de en los Centros de Actividad, en el tercio este de la región.

La zona oeste, de mala accesibilidad, se encuentra fragmentada por bandas de accesibilidad media por el efecto de las vías de comunicación que la atraviesan en especial de las dos principales. Así se observa el trazado de la N-630, por el Centro de la Región de Norte a Sur, y su impronta sobre la zona que atraviesa; lo mismo sucede con la zona próxima al recorrido de la $\mathrm{N}-\mathrm{V}$ hasta Badajoz.

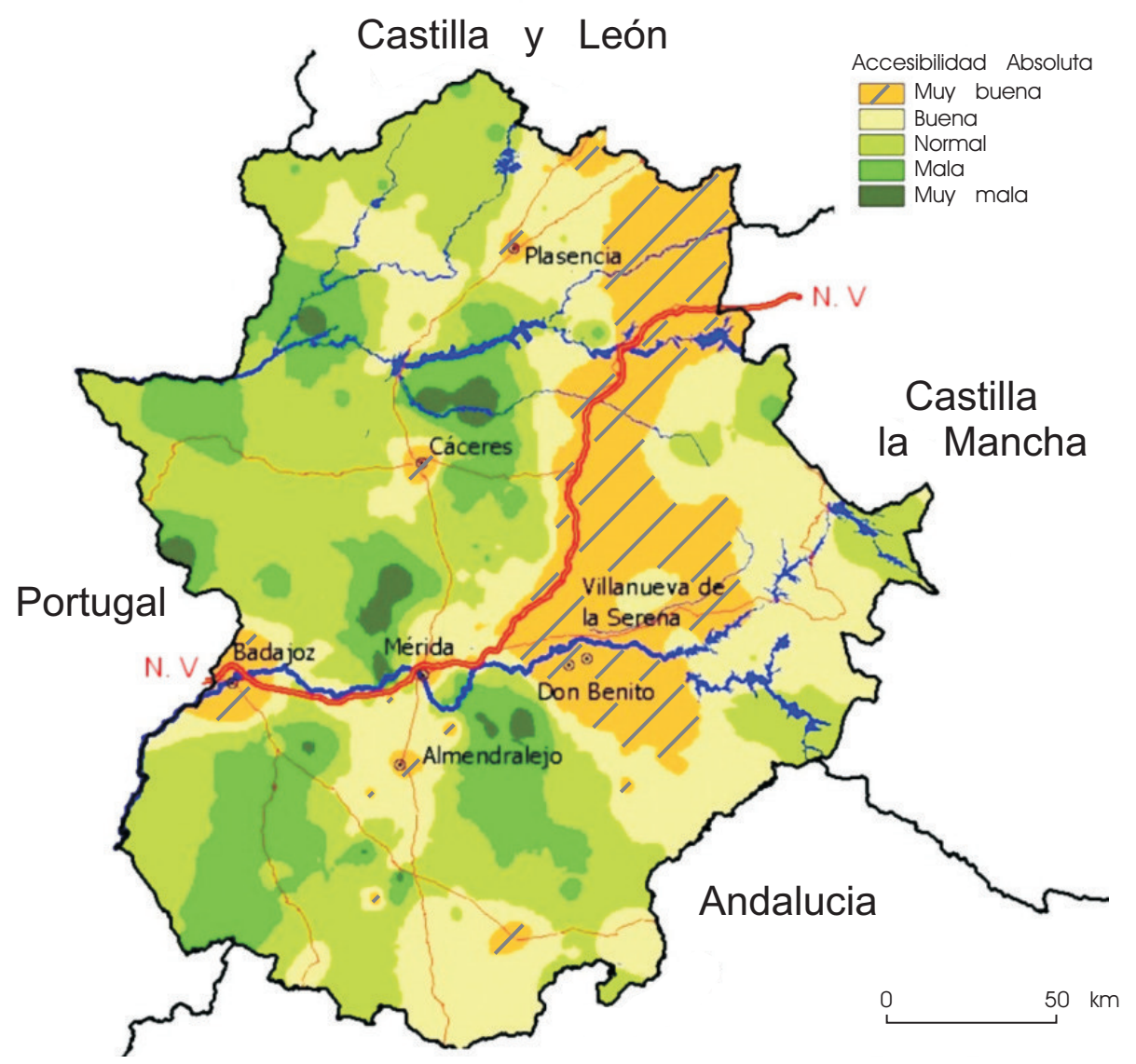

Fig. 3 - Accesibilidad relativa en Extremadura.

Fig. 3 - Relative Accessibility in Extremadura. 
Las zonas de muy mala accesibilidad relativa, al contrario que la accesibilidad absoluta, se encuentran ahora en zonas puntuales del interior de la región, generalmente. Zonas en las que la red de carreteras es de mala calidad o tiene un elevado índice de rodeo para poder sortear los cursos fluviales o las barreras orográficas, hasta acceder a los centros de actividad económica.

\section{TIEMPOS DE ACCESO MÍNIMO}

Este mapa (fig. 4) muestra el grado le lejanía a través de la red de carreteras a los Centros de Actividad Económica de Extremadura.

De esta manera podemos observar las zonas más periféricas del Norte de la región, la frontera con Portugal en Cáceres, en el Sur en los límites con las provincias de Sevilla y Huelva y destaca sobre todo la amplia zona del Este de Extremadura limitando con las provincias de Ciudad Real y Toledo que son las zonas más alejadas de los Centros de Actividad Económica.

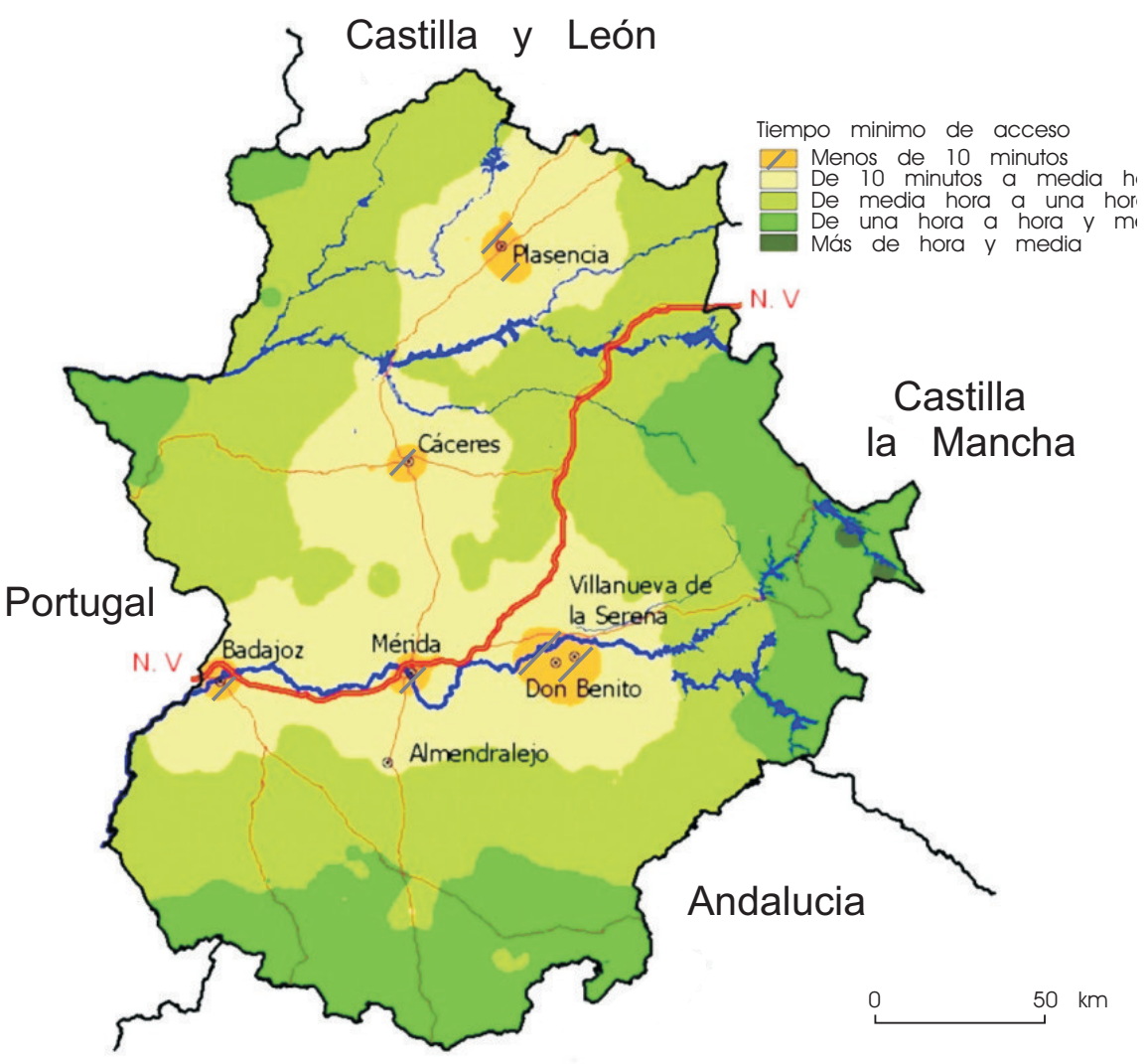

Fig. 4 - Tiempos de acceso mínimo en Extremadura.

Fig. 4 - Times of minimum access in Extremadura. 
El 90\% de la población de Extremadura reside a menos de una hora de desplazamiento de alguno de los núcleos principales. Las zonas más próximas como es lógico se ubican en las cercanías de los Centros de ctividad.

\section{MAPA DE ATRACCIÓN GRAVITATORIA}

En la figura 5 se pueden observar las áreas de atracción de los diferentes Centros de Actividad Económica. Se ven claramente las zonas de competencia entre los diferentes Centros de Actividad. Como consecuencia de la expresión utilizada las localidades sienten mayor atracción hacia las poblaciones más pobladas y más cercanas.

Así observamos una fuerte competencia en el eje del río Guadiana y en el centro de la provincia cacereña.

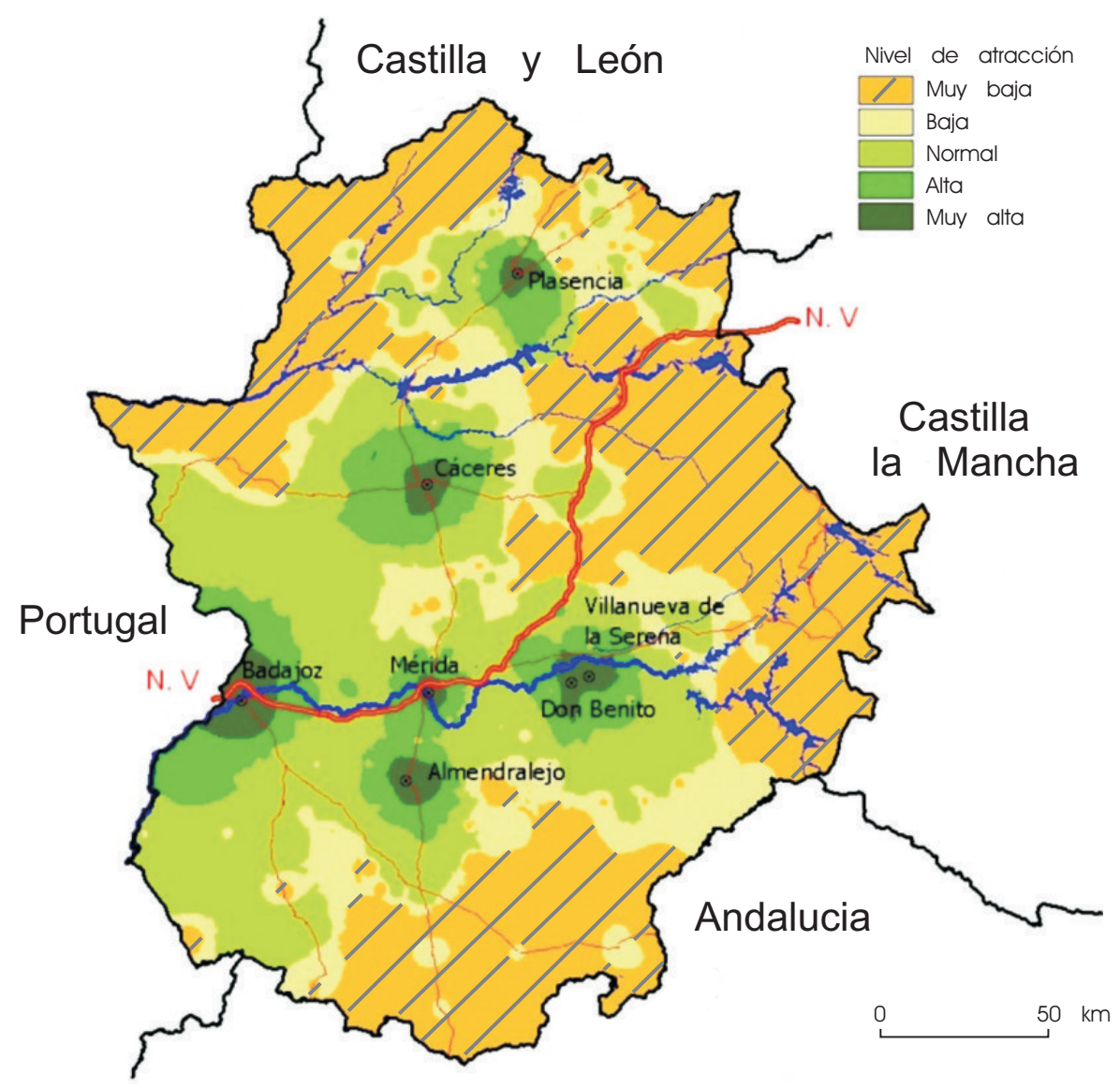

Fig. 5 - Atracción gravitatoria en Extremadura.

Fig. 5 - Gravitational pull in Extremadura. 
De igual forma vemos como la zona de atracción de la ciudad de Badajoz se extiende hacia el Norte y el Sur siguiendo la frontera portuguesa donde no tiene la competencia de otros Centros. La zona de atracción de Don BenitoVillanueva de la Serena se extiende hasta el límite con la provincia de Córdoba por el Sureste y hacia el valle del río Ruecas por el Noreste. La zona de atracción de Cáceres se alarga al oeste hacia Trujillo en la N-V. La zona de atracción de Plasencia se alarga al oeste hacia Coria y al este hacia Navalmoral de la Mata.

\section{CONCLUSIONES}

En lo que se refiere a los resultados del estudio sobre accesibilidad apuntamos lo siguiente:

a. Los resultados son un fiel reflejo de la realidad extremeña. Se observa una como el nivel del sistema de transportes es sinónimo del desarrollo de las diferentes zonas. Es decir el sistema de ciudades condiciona la red de transportes y viceversa, dado que la organización del territorio extremeño se basa sobre un sistema de asentamiento muy ligado a las potencialidades del medio natural;

b. Se muestran las zonas desfavorecidas y en retroceso de Extremadura. Son las zonas de frontera con poca densidad de habitantes los cuales deben realizar largos y caros desplazamientos para obtener bienes y servicios. Consiguientemente, la inadecuada red de transporte genera que las citadas zonas de mala accesibilidad absoluta queden cada vez más en posiciones ultra periféricas, lo que hace difícil su integración en el sistema de ciudades extremeñas. En este sentido, cabe apuntar que estas zonas remotas orientan su dependencia comercial hacia centros urbanos extrarregionales;

c. La red de transporte extremeña depende de las dos grandes carreteras nacionales que la atraviesan (N-630 y N-V). De hecho los Centros de Actividad Económica seleccionados se encuentran en ellas o próximos. Fuera de estas dos vías las infraestructuras son deficientes. Lo que muestra que no se ha realizado una planificación del transporte interno de Extremadura;

c. Las malas infraestructuras hacia Portugal muestran el efecto de la frontera sobre la red de carreteras extremeña. La «raya» se ha convertido a lo largo de los últimos siglos en un obstáculo tan importante o más que el medio físico para la cooperación entre las ciudades de uno y otro lado de la frontera, erigiéndose en un fondo de saco (donde todo acaba) que inhibía el desarrollo de esa zona y la aparición de ciudades, si exceptuamos el caso de Badajoz por su papel de plaza militar y capital provincial; 
e. Los factores fisiográficos, tanto de relieve sinuoso y abrupto como la retención de los cursos fluviales para almacenamiento de agua, se constituyen en elementos que determinan la disposición de los trazados de la red de carreteras. Se adivinan las barreras que suponen las zonas montañosas. Las sierras del Norte, Sierra de San Pedro en el centro y Sierra Morena en el sur se yerguen en barreras que aíslan los territorios intrarregionales y nos separan de otras comunidades autónomas. Los dos principales colectores fluviales, los ríos Tajo y Guadiana junto con sus embalses, por su discurrir transversal de este a oeste paralelos, fragmentan y dividen a la región en dos partes en su área central;

f. Se determinaría de una forma más precisa los tiempos de recorrido de los diferentes tramos de carretera introduciendo datos de las mismas que influyan en este tiempo tales como: intensidad del tráfico de vehículos, volumen de vehículos pesados, estado de conservación, ancho de la calzada, etc. Las travesías de los núcleos de población también suponen una resistencia al movimiento de vehículos y por tanto se podría asignar una impedancia a estos nodos en el SIG;

g. Para mejorar el modelo implementado, a los núcleos de población se les podría asignar otras características (distribución de los sectores productivos, equipamientos social y tecnológico, etc.) para realizar diferentes estudios económicos y sociales relacionados con las infraestructuras de transportes;

h. Como ya indicamos este estudio se ha realizado considerando a la red de transportes como un sistema aislado, lo cual no es cierto del todo, por esto en un estudio posterior habría que incluir las relaciones con las redes de transporte de España y Portugal. La nueva perspectiva europea hace que la frontera hispano lusa desaparezca como obstáculo infranqueable lo que conlleva cambios de accesibilidad a ambos de la frontera que será necesario estudiar y tener en cuenta;

i. El fin último de la investigación y de la evolución de un SIG es determinar el efecto de una acción antes que esta se ejecute. En el SIG implementado se podrían determinar el efecto que ciertas actuaciones sobre la red de carreteras producirían sobre la accesibilidad de una zona determinada tales como la mejora, construcción o corte de una carretera, la construcción de un puente o de un embalse, etcétera.

\section{BIBLIOGRAFÍA}

Caja de Ahorros y Pensiones de Barcelona (2002) - Anuario Económico de España 2002. La Caixa, Barcelona.

Gurría, J. L. (1992) - La Vía de la Plata como eje Vertebral en el Sistema de Transportes Cacereño. Cámara de Comercio e Industria de Cáceres, Cáceres: 22. 
Gutiérrez Puebla, J. e Monzón, A. (1993) - Accesibilidad a los Centros de Actividad Económica Antes y Después del Plan. Ciudad y Territorio, 1 (97), Madrid: 385-387.

Gutiérrez Puebla, J. et al. (1994) - Accesibilidad a los Centros de Actividad Económica en España. Revista de Obras Públicas, 3331, Madrid: 39-42.

Mora Aliseda, J. e Gutiérrez Gallego, J. A. (1998) - Nuevas Tecnologías Aplicadas a la Investigación Social. El caso de Extremadura. Mapping, Madrid: 18-26.

Potrykowski, M. e Taylor, Z. (1982) - Geografía del Transporte. Ariel, Barcelona: 11.

Ruíz Requena, A. (1992) - Sistemas de Transporte. Universidad de Granada, Granada: 14-15, 53.

SÁnchez Zabala, R. (1988) - Delimitación de Áreas de Influencia Urbana en Extremadura. Aplicación de un modelo de gravedad. Estudios territoriales, 27. Madrid: 88-90. 